\title{
Cloud Data Governance In-Light of the Saudi Vision 2030 for Digital Transformation
}

\author{
Majid Al-Ruithe and Elhadj Benkhelifa \\ Cloud Computing and Applications Research Lab \\ Staffordshire University \\ College Road, Stoke on Trent, UK \\ mrowathi@gmail.com, e.benkhelifa@staffs.ac.uk
}

\begin{abstract}
Digital transformation in Saudi Arabia is one of the core elements to achieve the goals of Saudi Vision 2030. In this paper, we argue that data governance plays a vital role for the success of this vision. This role is further emphasized when considering the country's appetite for emerging technologies such as cloud computing solutions. A thorough survey of literature shows that data governance in general and for cloud computing, more specifically, is under researched. This paper puts the Kingdom of Saudi Arabia Vision 2030 for digital transformation under a test. Supported by an empirical study, this paper provides an early warning of a highly likely failure if data governance is not integrated in the vision as a driver for digital transformation. An empirical survey, using a self-administered questionnaire, is conducted to explore and evaluate the status of data governance in the Saudi Arabia. The results of the study reveal that despite the wide recognition of the importance of data governance, especially for cloud computing, there is a consensus that this area is really in its infancy and almost absent from the Saudi organizations
\end{abstract}

Keywords-Data governance; Cloud computing ;Cloud data governance;Digital Transformation, Saudi vision 2030; Saudi Arabia.

\section{INTRODUCTION}

The volume of data used by organizations has increased dramatically in recent years, and playing a critical role in business operations [1]. Data can influence both operational and strategic decisions of any organization, hence governing this data is becoming ever more critical, [2]. Data governance has become an emerging subject in the information system (IS) field [3]. In the literature, most of researchers considered data governance as a promising approach for organizations to improve the quality of their data [4], [5]. Data governance is the embodiment of the processes, methods, tools and techniques to ensure that the organization has data that is of high quality, reliable and auditable [6]. In addition, the data governance is an essential part of an overall corporate governance strategy, and has to follow corporate governance principles[4]. A good data governance can help people within that organization to create a clear mission, achieve clarity, increase confidence of using the organizational data, establish accountabilities, maintain scope and focus, and define measurable successes [7], [8]. The literature argues that currently there is no single approach to the implementation of data governance program in all organizations [9]. Despite its significance, research on data governance is still in its infancy and needs more efforts [4], [9]. Regarding cloud data governance, one of the main reasons that impedes the wider adoption of the cloud computing model have been linked primarily to aspects related to data governance [10], [11]. While security shows to be the most cited challenge to cloud adoption, [12] show that $41 \%$ of the security problems in the cloud are related to governance and legal issues. Cloud governance is a new term in the IT field, and it is still under-developed [13], [14]. Microsoft defines cloud governance as "defining policies around managing the factors: availability, security, privacy, location of cloud services and compliance and tracking for enforcing the policies at run time when the applications are running". Data governance is considered as one of the most important aspects in cloud governance [10], [14]. Data governance program, built for on-premises IT infrastructure, cannot be deployed for a cloud infrastructure and service provisioning, which would require a completely new requirements, design and implementation [15], [16], [17], [14]. Undoubtedly, the area of cloud data governance is becoming a topic of the next coming decades [18], although, it is still under researched by both academia and industry due to its novelty [4], [9].

There are little studies reported on data governance for cloud services. Almost all existing work on data governance for cloud computing focus on the aspects of accountability and Interoperability [11], [13] [19-21]. With regards to the Saudi Arabia context, the government attaches high significance to egovernment and the transformation process that leads to its realization, in order to reduce costs, improve services, save time and increase effectiveness and efficiency across the public sector [22]. As a result, the government of Saudi Arabia has already started the process of implementing an e-government strategy. 'YESSER' has been the umbrella organization and the overall controller of all procedures related to e-government implementation in the Kingdom [23]. Moreover, in 2016 the Saudi government established the Saudi Arabia vision 2030, which includes the digital transformation program [24]. The public sector organizations involved in this vision, and the information communication technology will play a critical role to achieve Saudi vision 2030. However, for this vision to 
succeed, a data governance strategy needs to be in place, govern data, which plays a significant role in the planned digital transformation. Literature shows that there is almost an absence of study on data governance in general and more specific for cloud computing in Saudi Arabia. This study aims to contribute to the IS community by identifying the role of digital transformation to achieve the Saudi vision 2030, and present the current state of cloud data governance implementation in public sector organisations in Saudi Arabia by an empirical study. This paper is organised as follows: Section 2 presents an overview of the Saudi Arabia vision 2030; Section 3 presents the overview of the digital transformation. Section 4 describes the important of cloud data governance; and Section 5 presents the results of the empirical findings from KSA public sector. We conclude by discussing the results and addressing the limitations and recommendations for future work in the area in Section 6.

\section{SAUdi ARABIA VISION 2030: OVERVIEW}

The Kingdom of Saudi Arabia has recently launched the Saudi vision 2030 with the aim to build the best future for the country and its citizens, moving away, gradually from, Oil-dependent economy towards more diverse economy, including the digital economy [24]. However, the digital economy is underpinned by a strategy for digital transformation [26] throughout the whole kingdom, in all sectors. The vision has considered governance; Figure 1 depicts a high-level governance model, proposed as part of the Saudi vision 2030 by the Council of Economic and Development Affairs. This model aims at institutionalizing and facilitating the coordination of efforts among relevant stakeholders and effectively following-up progress for achieving Saudi Arabia's vision 2030 [24]. This model will encourage the public sector organization in Saudi Arabia to develop governance model to implement the digital transformation program, and to govern their digital data.

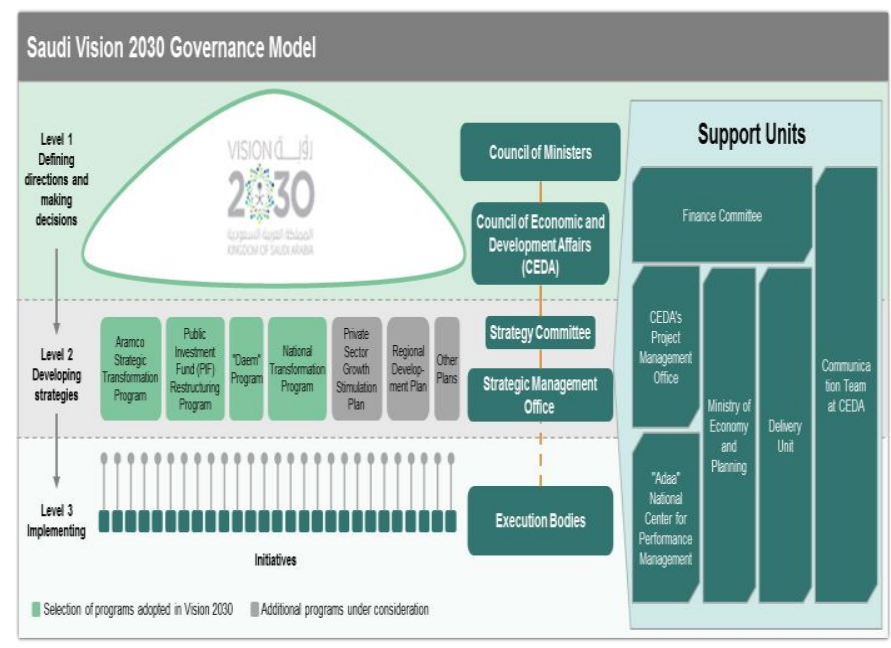

Fig 1. The governance model for achieving Saudi Arabia's vision 2030 [24].
Cloud computing is expected to be one of the main foundational enablers in future digital transformation projects and offers the scale and speed that is needed for businesses to focus on transformation; with a clear impact on the role of the IT department [27-30]. In Saudi vision 2030, the public sector organizations will lead to adopt cloud computing, thus that requires more efforts from these organizations to build a strong strategy to get control on their data when it moves to a cloud environment [31], [32]. Our previous published research on cloud computing adoption in Saudi Arabia [33] shows that the cloud computing is still an emerging paradigm in the country; it is not even in the agenda of the majority of public sector organizations [34]. Our research has also reconfirmed that the fear of loss of data governance is one of the main obstacles to adopt the cloud model. Hence, to achieve digital transformation by adopting cloud computing, designing and implementing effective data governance will play a key role for success.

\section{IMPORTANCE OF DATA GOVERNANCE FOR CLOUD COMPUTING}

Many organizations have become aware of the increasing importance of governing their data, to ensure confidentiality, integrity, quality and availability of business and customers' data [10], [35], [36]. There is no single approach to the implementation of data governance program in all organizations [37]. An effective data governance can help organizations to create a clear mission, achieve clarity, increase confidence of using the organizational data, establish accountabilities, maintain scope and focus, and define measurable successes [2], [9]. Moreover, many authors suggested that developing an effective data governance would lead to many benefits for organizations. These benefits are: enabling better decisionmaking, reduce operational friction, and protect the needs of data stakeholders as central to a governance program[18], [37]. In addition, other benefits include: training of management and staff to adopt common approaches to data issues, build standard, repeatable processes, reduce costs and increase effectiveness through coordination of efforts, and ensure transparency of processes [7], [18].

Data governance is often confused with data management [5], [12], [15]. The definition of data management provided by the Data Management Association (DAMA) is: "data management is the development, execution and supervision of plans, policies, programs and practices that control, protect, deliver and enhance the value of data and information assets". Data management in general focuses on the defining of the data element, how it is stored, structured, and moved. DAMA defines data governance as "the exercise of authority, control and shared decision-making (planning, monitoring and enforcement) over the management of data assets". Data governance is therefore, high-level planning and control over data management [38]. Authors of [2] have also argued that data governance is different from data management; that data governance complements data management, but does not replace it. Authors of [7] defined data governance as "a system of decision rights and accountabilities for information-related 
processes, executed according to agreed-upon models which describe who can take what actions with what information, and when, under what circumstances, using what methods". While authors of [39] defined data governance as "specifies the framework for decision rights and accountabilities to encourage desirable behavior in the use of data. To promote desirable behavior, data governance develops and implements corporatewide data policies, guidelines, and standards that are consistent with the organization's mission, strategy, values, norms, and culture".

Some other researchers or practitioners may also confuse between IT governance and dada governance. IT governance is a much more mature area with the first publications on the topic released about four decades ago [40], [41], while data governance is still under-researched. Organizations with mature IT governance practices tend to have stronger alignment between IT and business [42]. Authors of [43] argued that organizations should get responsibility for data out of the IT department. Besides of IT governance, data governance also has a significant role in aligning the organization's business[43]. Data governance can be used to solve assortment of business issues related to data and information[18], [39], [44]. Authors of [40] also argued that a data governance model helps organizations to structure and document the accountabilities for their data quality. Authors of [45] have explicitly demonstrated that data governance is different from IT governance in principal and practices. In principal, data governance is designed for the governance of data assets, while IT governance makes decisions about IT investments, IT application portfolio, and the IT projects portfolio. In practice, IT governance is designed primarily around an organization's hardware and applications not its data. Authors of [46] argued that the enterprise-wide data strategy and governance are important for organizations and they need these to achieve competitive advantage. In addition, all existing sources have hitherto only addressed data governance, partially[40]. The fact that organizations need to take into account many aspects into consideration when implementing data governance has been neglected so far [47]. Moreover, some researchers show that if organizations who do not implement an effective data governance, can quickly lose any competitive advantage[7], [46]. Authors of [41] illustrated that working without a proper data governance programme is analogous to an organization allowing each department and each employee to develop, for instance, its own financial chart of accounts. Data governance requires the involvement and commitment of all staff with full sponsorship by the management and senior-level executive sponsorship in any organization [48], [49].

Regarding the role of the cloud data governance in the digital transformation, it mitigates the risks associated with data in the cloud and it can offer valuable costs savings, protecting the organizations from the potential costs of managing a security breach, for instance. Penalties for improper use and storage of data can be hefty, not to mention the damage a breach can do to your customer relationships.

\section{REALITY OF DATA GOVERNANCE IN KSA}

As mentioned above Digital transformation in Saudi Arabia is one of the core elements to achieve the goals of the Saudi Vision 2030. In this paper, we argue that data governance plays a vital role for the success of this vision. This role is further emphasized when considering the country's appetite for the cloud-computing paradigm. This paper puts the Kingdom of Saudi Arabia Vision 2030 for digital transformation under a test. Supported by an empirical study, this paper provides an early warning of a highly likely failure if data governance is not integrated in the vision as a driver for digital transformation. An empirical survey, using a self-administered questionnaire, is conducted to explore and evaluate the status of data governance in the Saudi Arabia. A total of 429 questionnaires were distributed between $13 / 11 / 2016$ to $31 / 1 / 2017$, of which 292 responses were received from the main public sector representatives. The response rate of almost $70 \%$ is considered adequate to draw reliable conclusions about the state of the art of cloud data governance in Saudi Arabia.

\section{A. Sample Demography and Charactristics}

Table 1 provides a general overview of the demographic characteristics of the participants, such as organization size, organization sector, role in the organization, number of IS specialists, participant's experience, data governance experience and cloud computing experience.

Table 1. The demographic characteristics of the participants.

\begin{tabular}{lll}
\hline \multicolumn{1}{c}{ Characteristics } & Frequency $(n)$ & Percent $(\%)$ \\
\hline Organisation Size: & & \\
More than 5000 employees & 69 & $33.50 \%$ \\
5000-1000 employees & 64 & $31.07 \%$ \\
1000-500 employees & 31 & $15.05 \%$ \\
Less than 500 employees & 42 & $20.39 \%$ \\
Organization Sector: & & \\
Military & 39 & $18.93 \%$ \\
Financial Services & 31 & $15.05 \%$ \\
Healthcare & 33 & $16.02 \%$ \\
Education & 42 & $20.39 \%$ \\
Telecommunication \& & 25 & $12.14 \%$ \\
Information Technology & & \\
Other & 36 & $17.48 \%$ \\
Role in the organization: & & \\
CEO & 10 & $4.85 \%$ \\
CIO & 15 & $7.28 \%$ \\
Vice President & 7 & $3.40 \%$ \\
IT Manager & 42 & $20.39 \%$ \\
Cloud Manager & 6 & $2.91 \%$ \\
IT Specialist & 59 & $28.64 \%$ \\
Other & 67 & $32.52 \%$ \\
& &
\end{tabular}


Number of IS Specialists:

$\begin{array}{lll}\text { Less than } 10 \text { Specialists } & 66 & 33.50 \% \\ \text { Between } 11 \text { and } 20 & 21 & 10.66 \% \\ \text { Specialists } & 28 & 14.21 \% \\ \text { Between } 21 \text { and } 30 & 19 & 9.64 \% \\ \text { Specialists } & 8 & 4.06 \% \\ \text { Between } 31 \text { and } 40 & 55 & 27.92 \% \\ \text { Specialists } & & \end{array}$

Between 41 and 50

Specialists

Over than 50 Specialists

Participant's experience:

More than 10 years

Between 5 and 10 years

Between 2 and 5 years

Less than 2 years

Participants'data governance experience:

$\begin{array}{lll}\text { No experience and } & 45 & 22.84 \% \\ \text { Knowledge } & 52 & 26.40 \% \\ \text { No experience but good } & 30 & 15.23 \% \\ \text { Knowledge } & 26 & 13.20 \% \\ \text { More than } 10 \text { years } & 32 & 16.24 \% \\ \text { Between } 5 \text { and } 10 \text { years } & 12 & 6.09 \% \\ \text { Between } 2 \text { and } 5 \text { years } & & \\ \text { Less than 2 years } & & \\ \text { Participants' cloud } & & \\ \text { computing experience: } & & 20.30 \% \\ \text { No experience and } & 40 & 33.50 \% \\ \text { Knowledge } & 66 & 14.72 \% \\ \text { No experience but good } & 29 & 16.24 \% \\ \text { Knowledge } & 32 & 11.68 \\ \text { More than } 10 \text { years } & 23 & 3.55 \\ \text { Between } 5 \text { and } 10 \text { years } & 7 & \\ \text { Between } 2 \text { and } 5 \text { years } & & \\ \text { Less than } 2 \text { years } & & \end{array}$

\section{B. Cloud Data Governance}

Respondents were asked to specify their understanding of data governance, importance of the cloud data governance, and the current state of the cloud data governance in their organizations.

\section{- Data Governance Definition}

There isn't an official definition about a data governance in the literature, this study adopts a popular definition that has been defined by the data governance institute (DGI): "the framework for decision rights and accountabilities to encourage desirable behavior in the use of data, to promote desirable behavior, data governance develops and implements corporate-wide data policies, guidelines, and standards that are consistent with the organization's mission, strategy, values, norms, and culture" [49]. This definition was given to the participants to seek their views. The approach taken by Kooper et al. was adopted to measure information governance definition status in organization based on IBM definition [50]. The responses to the data governance definition question were classified as follows:

- The definition is essentially the same in my organization.

- My organization have another definition.

- My organization have no definition for data governance.

- Don't Know.

Respondents were asked to describe areas where their organization definition of data governance differed from that of DGI (Figure 2). Of those who answered this question, most of the respondents reported that their organizations have no definition for data governance $32.04 \%$, followed by $28.64 \%$ were the definition is essentially the same in my organization. Next, $25.73 \%$ reported 'don't know', and $13.59 \%(\mathrm{n}=28)$ said their organization have another definition.

\section{Data Governance Definition}

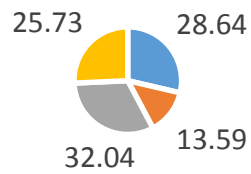

- The definition is essentially the same in my organisation

- My organisation have another definition

- My organisation have no definition for data governance

- Don't Know

Fig 2. The answer of the data governance definition.

\section{- Importance of Implementing Cloud Data Governance Program}

To identify the importance of implementing cloud data governance program in Saudi public sector organization, the survey asked participants to indicate the importance of implementing cloud data governance in their organizations (Figure 3). The responses were classified as follows: $72.33 \%$ reported that it is important, $22.33 \%$ pf respondents were not sure and only $5.34 \%$ of the respondents thought it is not important. 


\section{Importance of Implementing Cloud Data Governance Program}

\author{
22.33

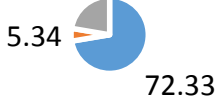

- Important - No important $\quad$ Don't Know

Fig 3. Results of the questionnaire on the Importance of implementing cloud data governance program.

\section{- Current State of Cloud Data Governance Program}

The current state of cloud data governance refers to the extent of cloud data governance program implementation state public sector organizations in Saudi Arabia. For this investigation, we have adopted study adopted Kooper et al. approach to measure information governance status in IBM organization [50]. The current state of the cloud data governance program implementation was measured using closed question (Yes, No, Don't Know).

The results (Figure 5) show that the majority of the respondents $(67.48 \%)$ do not have any form of cloud data governance program in their organizations, and $18.93 \%$ were not sure 'don't know'. About $14 \%$ of participants believe that they have some form of cloud data governance programme, but limited. Figure 4 shows the distribution of responses on the question on the current state of the cloud data governance implementation.

\section{The current state of cloud data governance program}

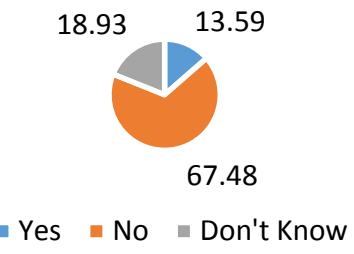

Fig 4. The current state of the cloud data governance implementation in Saudi Arabia Public Sector.

Participants, who answered with 'NO', will need to answer a sub-question in order to investigate the organizations' plan for implementing cloud data governance; $50 \%$ of the respondents reported it is actually not discussed and $18 \%$ stated they do not intend to implement cloud data governance program at this time. Only $15 \%$ reported that they intend to implement cloud data governance program in the next 2 years. In addition, $7.19 \%$ reported that they intend to implement cloud data governance program in the next 1 year, and $6.47 \%$ were not aware of any plans to do so. On $3.6 \%$ or participants had the intention to implement cloud data governance program in the next 6 months.

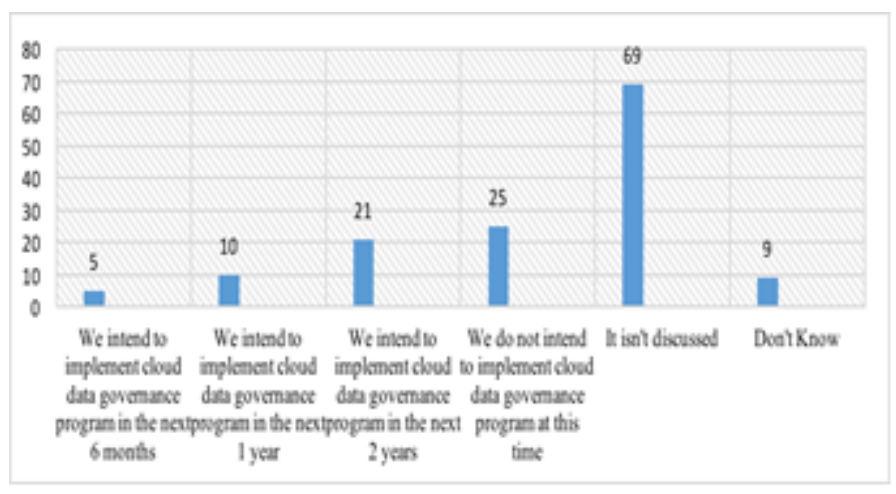

Fig 5. The Saudi participating organizations' plan for implementing cloud data governance program.

\section{Conclusions}

This study aimed at putting the Saudi vision 2030 for digital transformation under a reality check by investigating what we argue to be a fundamental pillar for the success of the vision, that the state of the art of Data Governance, especially, for cloud computing in the country. This research supports the vision with new results, which can help its implementation with early considerations of aspects related to Data Governance. The study focused on three perspectives: the data governance definition, important of cloud data governance implementation, and the current state of cloud data governance. The sample demography for the study, given in Table 1 above, is considered a good representation to draw realistic conclusions on the current state. More than a third of respondents were from organizations with more than 5,000 employee, where 56\% covered three vital sectors, Education, Military and Healthcare. The results of this study confirm that the cloud data governance is still in its infancy. Up to $86 \%$ of KSA's public sector organizations currently do not implement any form of cloud data governance. Out of the remaining 14\%, who reported to have some forms of cloud data governance, the Military sector seems to be in a leading position, followed by the Telecommunication \& Information Technology, and the Education Sectors. The lack of knowledge and awareness about the importance of cloud data governance, shown by the respondents, even at senior position, raises a challenging future for success and sustainability of the Saudi vision 2030 for digital transformation. The main recommendation from this research emphasises on the importance of developing a comprehensive and holistic framework for data governance in general and for emerging technologies, in particular, which will support the implementation of the plans for the envisaged digital transformation in Saudi Arabia 


\section{Acknowledgment}

The authors would like to thank the Government of Saudi Arabia for supporting this research through a $\mathrm{PhD}$ scholarship. The authors also extend their gratitude to Dr. Abdullah Al-Faifi who helped us with distributing the questionnaires.

\section{REFERENCES}

[1] S. Sakr, a Liu, D. M. Batista, and M. Alomari, "A Survey of Large Scale Data Management Approaches in Cloud Environments," Commun. Surv. Tutorials, IEEE, vol. 13, no. 3, pp. 311-336, 2011.

[2] I. B. M. Master and D. Management, "Data Governance for Master Data Management," Data Manag., pp. 67-86, 2007.

[3] I. Alhassan, D. Sammon, and M. Daly, "Data governance activities: an analysis of the literature," J. Decis. Syst., vol. 25 , no. S1, pp. 64-75, 2016.

[4] K. Wende, "Data Governance Defining Accountabilities for Data Quality Management," D’Atri, Alessandro (Hrsg.), 2007.

[5] B. Otto, "A Morphology of the Organisation of Data Governance," ECIS 2011 Proc., p. 272, 2011.

[6] P. Russom, "Data Governance strategies: Helping your organization comply , transform , and integrate," Dato Warehous. Inst., 2008.

[7] Z. Panian, "Some Practical Experiences in Data Governance," World Acad. Sci. Eng. Technol., pp. 939 - 946, 2010.

[8] X. Fu, A. Wojak, D. Neagu, M. Ridley, and T. Kim, "Data governance in predictive toxicology: A review," $J$ Cheminform., vol. 3, no. 1, p. 24, 2011.

[9] C. Begg and T. Caira, "Exploring the SME Quandary: Data Governance in Practise in the Small to Medium-Sized Enterprise Sector," Electron. J. Inf. Syst. Eval., vol. 15, no. 1, pp. 3-13, 2012.

[10] S. Groß and A. Schill, "Towards user centric data governance and control in the cloud," Lect. Notes Comput. Sci. (including Subser. Lect. Notes Artif. Intell. Lect. Notes Bioinformatics), vol. 7039 LNCS, pp. 132-144, 2012.

[11] B. Mary, P. Mccarthy, and S. Hill, "Cloud Adoption Points to IT Risk and Data Governance Challenges," Directorship, no. September 2010, pp. 2011-2011, 2011.

[12] S.-H. Kim and I.-Y. Lee, "Study on User Authority Management for Safe Data Protection in Cloud Computing Environments," Symmetry (Basel)., vol. 7, pp. 269-283, 2015.

[13] A. S. Bachelor and I. Technology, "Lewam Woldu Cloud Governance Model and Security Solutions for Cloud Service Providers," no. May, 2013.

[14] S. Saidah and N. Abdelbaki, "A new cloud computing governance framework," CLOSER 2014 - Proc. 4th Int. Conf. Cloud Comput. Serv. Sci., pp. 671-678, 2014.

[15] W. Eckerson, "Creating an Enterprise Data," Tech Target, no. June, pp. 1-39, 2011

[16] N. Alkhater, G. Wills, and R. Walters, "Factors Influencing an Organisation's Intention to Adopt Cloud Computing in Saudi Arabia," 2014 IEEE 6th Int. Conf. Cloud Comput. Technol. Sci., pp. 1040-1044, 2014.

[17] G. Eugene, "Cloud Computing Models," MIT Eng. Syst. Div., no. January, 2013.

[18] P. De Hert and V. Papakonstantinou, "Three Scenarios for International Governance of Data Privacy: Towards an International Data Privacy Organization, Preferably a UN
Agency?," I/S A J. Law Policy Inf. Soc., vol. 9, pp. 272-324, 2013

[19] M. Felici, T. Koulouris, and S. Pearson, "Accountability for Data Governance in Cloud Ecosystems," 2013 IEEE 5th Int. Conf. Cloud Comput. Technol. Sci., pp. 327-332, 2013.

[20] Madhuri C., "Cloud Information Accountability Frameworks for Data Sharing Inin Cloud," IOSR J. Comput. Eng., vol. 13, pp. 93-96, 2013

[21] S. Pearson, V. Tountopoulos, D. Catteddu, M. Sudholt, R. Molva, C. Reich, S. Fischer-Hubner, C. Millard, V. Lotz, M G. Jaatun, R. Leenes, C. Rong, and J. Lopez, "Accountability for cloud and other future Internet services," CloudCom 2012 - Proc. 2012 4th IEEE Int. Conf. Cloud Comput. Technol. Sci., pp. 629-632, 2012.

[22] S. Salem Basamh, H. A. Qudaih, and M. Adam Suhaimi, "EGovernment Implementation in the Kingdom of Saudi Arabia: An Exploratory Study on Current Practices, Obstacles \&amp; Challenges," Int. J. Humanit. Soc. Sci., vol. 4, no. 2, pp. 296300, 2014

[23] R. Sharma, A. Sharma, and U. S. Pandey, "E - Governance : A Successful Implementation of Government Policies using Cloud Computing," Int. Conf. Web Servivces Comput., pp. 27-29, 2011

[24] S. V. 2030, "Saudi Vision 2030," 2017. [Online]. Available: http://vision2030.gov.sa/en. [Accessed: 05-Jul-2017].

[25] A. Abu-Musa, "Information security governance in Saudi organizations: an empirical study," Inf. Manag. \&amp; Comput. Secur., vol. 22, no. 3, pp. 235-250, 2010.

[26] IDC, "Digital Transformation (DX): An Opportunity and an Imperative," 2015.

[27] W. Zeng, Y. Zhao, K. Ou, and W. Song, "Research on cloud storage architecture and key technologies," Proc. 2nd Int. Conf. Interact. Sci. Inf. Technol. Cult. Hum. - ICIS '09, pp. 1044-1048, 2009

[28] J. Gubbi, R. Buyya, and S. Marusic, "Internet of Things ( IoT ): A Vision , Architectural Elements , and Future Directions," Futur. Gener. Comput. Syst., vol. 29.7, no. 29.7, pp. 1-28, 2013.

[29] S. P. Ahuja and B. Moore, "A Survey of Cloud Computing and Social Networks," Netw. Commun. Technol., vol. 2, no. 2 pp. 11-16, 2013.

[30] P. Mell and T. Grance, "The NIST Definition of Cloud Computing Recommendations of the National Institute of Standards and Technology," Nist Spec. Publ., vol. 145, p. 7, 2011

[31] A. Osman, S. M. Abdalrahman, and A. Elgelany, "Proposed Academic Cloud Computing for Saudi Universities and Higher Institutes," 2013 Fourth Int. Conf. e-Learning "Best Pract. Manag. Des. Dev. e-Courses Stand. Excell. Creat., pp. 113-119, 2013.

[32] T. H. Noor, "Usage and technology acceptance of cloud computing in Saudi Arabian Universities," Int. J. Softw. Eng. its Appl., vol. 10, no. 9, pp. 65-76, 2016.

[33] M. Al-Ruithe, E. Benkhelifa, and K. Hameed, "Current State of Cloud Computing Adoption - An Empirical Study in Major Public Sector Organizations of Saudi Arabia (KSA)," Procedia Comput. Sci., vol. 110, pp. 378-385, 2017.

[34] A. Youssef and M. Alageel, "A Framework for Secure Cloud Computing," Int. J. Comput. Sci., vol. 9, no. 4, pp. 487-500, 2012

[35] L. Hunter, "Tools for Cloud Accountability: A4Cloud Tutorial," 2015. [Online]. Available: http://www.a4cloud.eu/node/362. [Accessed: 04-Nov-2015].

[36] The Data Governance Institute, "Definitions of Data 
Governance," 2015. [Online]. Available: http://www.datagovernance.com/adg_data_governance_defin ition/. [Accessed: 16-Feb-2015].

[37] P. Statement, T. Public, and R. Officer, "Institutional Data Governance Policy,” pp. 1-12, 2009.

[38] ISACA.org, "ISACA," 2016. [Online]. Available: https://www.isaca.org/Pages/default.aspx. [Accessed: 23Mar-2016].

[39] E. Niemi, "Designing a Data Governance Framework," p. 14, 2011.

[40] W. V. G. S De Haes, "Practiices in IT Governance and Business/IT alignment," Inf. Syst. Control, vol. 2, pp. 1-6, 2008.

[41] A. Neff, M. Schosser, S. Zelt, F. Uebernickel, and W. Brenner, "Explicating performance impacts of it governance and data governance in multi-business organisations," Australas. Conf. Inf. Syst., pp. 1-11, 2013.

[42] K. Weber, B. Otto, and H. Osterle, "One Size Does Not Fit All - A Contingency Approach to Data Governance," ACM J. Data Inf. Qual., vol. 1, no. 1, pp. 4:1-4:27, 2009.

[43] K. Wende, "A Model for Data Governance - Organising Accountabilities for Data Quality Management," Corp. Gov., pp. 417-425, 2007.

[44] K. Weber, L. Cheong, B. Otto, and V. Chang, "Organising Accountabilities for Data Quality Management-A Data Governance Case Study.," Data Warehous., pp. 347-362, 2008.
[45] S. Rajendran, "Organizational Challenges in Cloud Adoption and Enablers of Cloud Transition Program," Mit, vol. 145, no. May, pp. 1-49, 2013.

[46] B. R. Prinzo and T. P. Group, "Developing a Strategy for your Data Governance Initiative," no. July, pp. 15-17, 2012.

[47] D. Catteddu and G. Hogben, "The European Network and Information Security Agency ( ENISA ) is an EU agency created to advance This work takes place in the context of ENISA' $s$ Emerging and Future Risk programme . C ONTACT DETAILS: This report has been edited by," Computing, vol. 72, pp. 2009-2013, 2009.

[48] B. B. Fleissner, K. Jasti, J. Ales, and R. Thomas, "The Importance of Data Governance in Healthcare," pp. 1-11, 2014.

[49] G. Thomas, "How to use the DGI data governance framework to configure your program," Data Gov. Inst., p. 17, 2009.

[50] E. Kooper, M., Maes, R., and Roos Lindgreen, "Information Governance as a Holistic Approach to Managing and Leveraging Information Prepared for IBM Corporation," Int. J. Inf. Manage., vol. 31, 2011. 